\title{
Dettmeyer/Verhoff/Schütz, Forensic Medicine: Fundamentals and Perspectives
}

\author{
Springer, Heidelberg Dordrecht London New York, 2014, 584 pp., \\ 379 figs. Hard cover (ISBN: 978-3-642-38817-0)
}

\author{
Daniel Wittschieber
}

Published online: 7 February 2014

(C) Springer-Verlag Berlin Heidelberg 2014

The present work represents the first comprehensive English language state-of-the-art textbook by German forensic scientists, covering all important aspects of modern legal medicine and forensic pathology.

The book consists of 30 concisely written and well-structured chapters integrating an established knowledge together with the latest developments published in English and German specialist literature. Commencing with detailed considerations about the external postmortem examination and death certification that most physicians are confronted with, the authors then proceed with the more specific topics arising during the further investigation of human bodies such as autopsy, exhumation, and identification. The next chapters are dedicated to the classical findings in forensic traumatology needed for the autopsy room. Wherever possible, the current state of forensic histopathology is presented. The subsequent chapters address the wide field of clinical forensic medicine in living individuals. Infanticide/ neonaticide, traffic medicine, forensic DNA analysis, forensic osteology, and the expanding field of forensic radiology represent the next parts. After dealing with sudden unexpected natural deaths, torture, forensic psychopathology, medical malpractice, as well as forensic alcohology and toxicology, the book ends with several appendixes providing numerous reference values and body charts for routine practical work.

The layout is very clear, and all information are easily accessible. In addition, the text is abundantly and colorfully illustrated with high-quality photographs, systematic diagrams, and innovative drawings. Clearly structured tables help in the understanding of complex aspects. To demonstrate forensic medicine as a discipline of special and unique cases, interesting case studies are presented at the beginning of most chapters. For those who want to know more, a list of selected references for further reading is provided in the end of each chapter.

R. B. Dettmeyer is not only a board-certified physician in two medical disciplines, general pathology and legal medicine, he also studied law. Equipped with this interdisciplinary experience, Professor Dettmeyer is predestined to be an author of a medicolegal textbook for international readership. M. A. Verhoff and H. F. Schütz optimally complement his expertise by contributing an ample experience and specific knowledge in their fields of science. Professor Verhoff's work is predominantly focused on forensic anthropology, osteology, and radiology, whereas Professor Schütz' scientific home is the forensic toxicology. Together, these three experts cover the whole profession of legal medicine.

"Forensic Medicine: Fundamentals and Perspectives" can be recommended to medical students and residents of legal medicine in particular. Moreover, every physician every physician involved in forensic issues as well as police officers, attorneys, or judges who are interested in understanding daily medicolegal case work in more detail will benefit from this new and internationally oriented compendium.
D. Wittschieber $(\bowtie)$

Institute of Legal Medicine, University Hospital Münster,

Röntgenstraße 23, 48149 Münster, Germany

e-mail: daniel.wittschieber@ukmuenster.de 